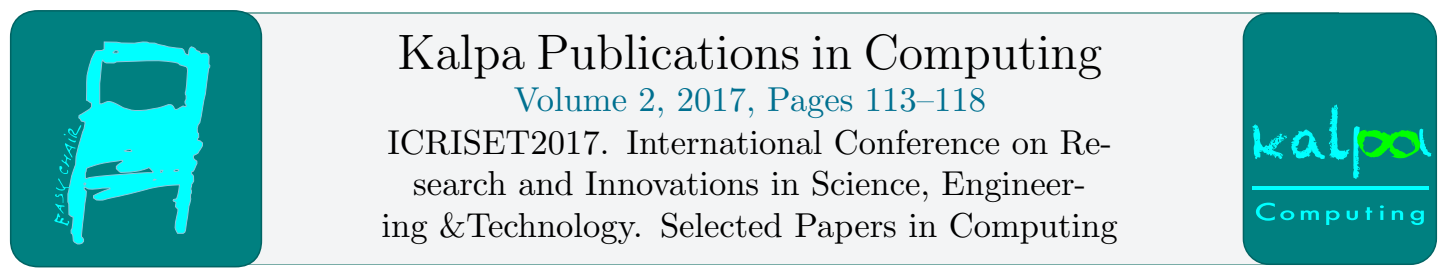

\title{
Flower Classification using Texture and Color Features
}

\author{
Riddhi H. Shaparia ${ }^{1}$, Dr. Narendra M. Patel ${ }^{2}$ and Prof. Zankhana H. Shah ${ }^{3}$ \\ ${ }^{1}$ PG student, ${ }^{2}$ Associate Professor, ${ }^{1,2}$ Computer Engineering Department \\ ${ }^{3}$ Head of Information Technology Department \\ 1,2,3 Birla Vishvakarma Mahavidyalaya, V.V.Nagar, Anand, India \\ ${ }^{1}$ shapariariddhi@gmail.com, ${ }^{2 n m p a t e l @ b v m e n g i n e e r i n g . a c . i n ~}$ \\ ${ }^{3}$ Zankhana.shahe bvmengineering.ac.in
}

\begin{abstract}
In this research paper, we have used texture and color features for flower classification. Standard database of flowers have used for experiments. The preprocessing like noise removal and segmentation for elimination of background are apply on input images. Texture and color features are extracted from the segmented images. Texture feature is extracted using GLCM (Gray Level Co-occurrence Matrix) method and color feature is extracted using Color moment. For classification, neural network classifier is used. The overall accuracy of the system is $95.0 \%$.
\end{abstract}

\section{Introduction}

This paper contains information about flower classification system using texture and color features. Digital image processing is handy in extracting useful information from different images having different background appearance. By using image processing techniques, we can do various task like classification, clustering, image recognition, pattern or character recognition and object detection from an images or videos. Image processing system is a sequence of operation on the images to enhance quality of the images or improve the imperfections of the images [12]. Creating a flower classification system is a difficult because of large intra-class variation present and small inter-class variation present among different classes [6]. The dataset of flowers contains the flower images are taken in natural environment where the shine of light changes with the time and weather [6]. Flower classification is an interesting and challenging problem.

Flower classification has various applications, as it can be helpful in flower searching for patent analysis and in floriculture. The floriculture industry consist of flower trade means selling and buying flowers, bulb and seed production, nursery and potted plants and extraction of essential oil from flowers. In above cases, automation of the classification of flower is necessary [1]. The other application of 
classification of flower is digital library, search engine which queries images based on text or keywords [13], gardening, Ayurveda treatment and CBIR (Content-based image retrieval).

\section{Related Work}

Dr.S.M.Mukane and Ms.J.A.Kendule [1] they discussed about the classification of flower based on texture feature. The method used for feature extraction is DWT (Discrete Wavelet Transform) and GLCM (Gray Level Co-occurrence Matrix). For flower classification, ANN (Artificial Neural Network) is used. However, they used only 50 images for classification, 10 images from each class. Very less dataset is used. Tanakorn Tiay, Pipimphorn Benyaphaichit, and Panomkhawn Riyamongkol [2] explored the concept of the recognition of flower. For classification of flowers, color and edge characteristics of flower images are used. To derive the color characteristics and edge characteristics, which are acquired through Hu's seven-moment algorithm, histogram is used. For classification, they take K-nearest neighbor. The accuracy of the system is more than $80 \%$. However, the problem is that similar color flower and similar shape flower has less accuracy. Soon-Won Hong and Lynn Choi [4] described an approach focused on automatic flower recognition using edge and color based contour detection. In pre-processing, the system performs morphological transformation and smoothing for noise removal from image. Then edge based contour and color based contour is detected, edge based contour is detected using first derivative, Sobel differential operation is used. Then to classify its color groups, K-means clustering is used. And for contour shapes, history matching is used. Anelia Angelova, Shenghuo Zhu and Yuanqing Lin [9] discussed about flower recognition for large-scale subcategory. They briefly describe image segmentation algorithm. Laplacian operator is used for segmentation. Support Vector Machine (SVM) is used for classification. D S Guru, Y. H. Sharath and S. Manjunath [6] they discussed about the automatic classification of flower images using K-nearest neighbor classifier. The images are segmented using threshold based segmentation algorithm. Then, the texture feature are extracted from an images using Gray Level Co-occurrence Matrix (GLCM), Gabor or combination of both. Then by using k-nearest neighbor classification is performed.

\section{The flower Classification System}

\subsection{Overview of the System}

The overview of the flower classification system using texture and color features are shown in fig. 1.

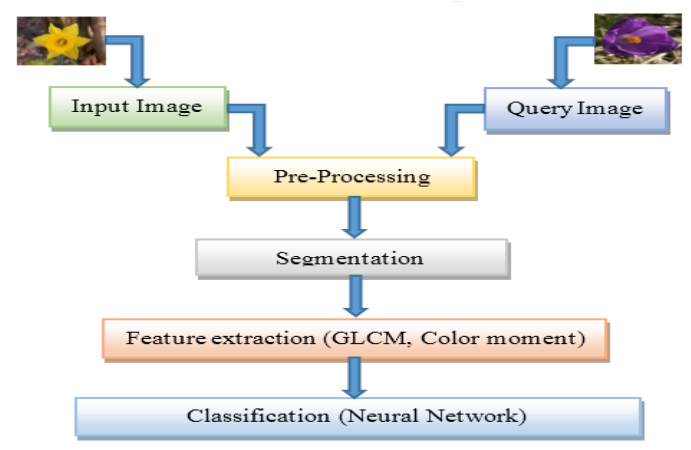

Figure 1 Block diagram for flower classification 
The input image is taken from dataset. Here the dataset used for classification is oxford 17 category flower dataset [13]. The input image is pre-processed for the noise removal and background elimination. Threshold is determined using Otsu's method. It is applied on image to divide image into foreground and background for segmentation. The texture feature is extracted using GLCM [1] [7] method, color feature is extracted using Color moment and classified by the neural network

\subsection{Pre-processing and Segmentation}

This section discuss about the pre-processing and segmentation techniques to make the image for further processing [2].
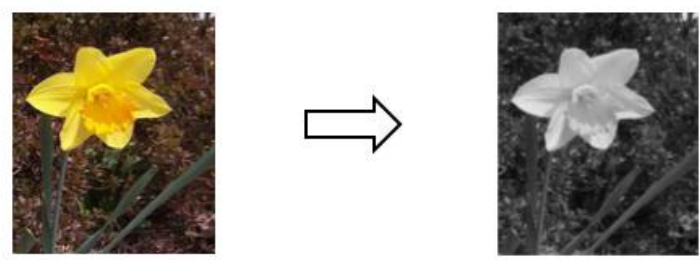

Figure 2 Conversion of color image into grayscale

First, for speedy processing the input image is resize to half. Then for segmentation convert the color image into grayscale as shown in fig. 2. By using threshold, the grayscale image is converted into binary image as shown in fig. 3 .
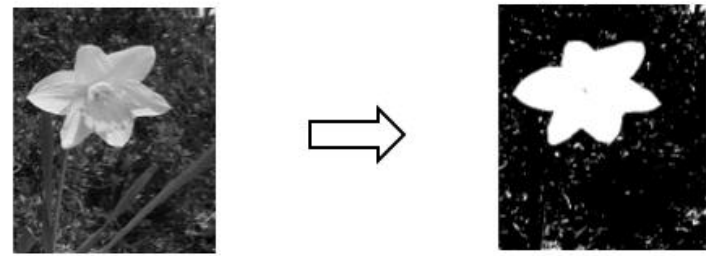

Figure 3 Conversion of grayscale image into binary image using threshold

However, this binary image contains noise; hence, we have to remove the noise. By using maximum connected component on binary image, we get the binary image without noise in which foreground is white and background is black. Create the bounding box around the foreground, as shown in fig. 4 .
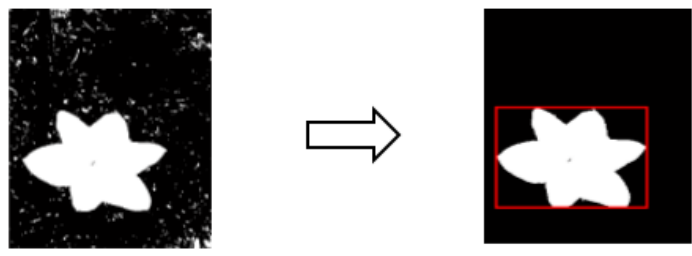

Figure 4 Binary image with bounding box

After finding the bounding box, flower is segmented from the original image as shown in fig. 5 . 

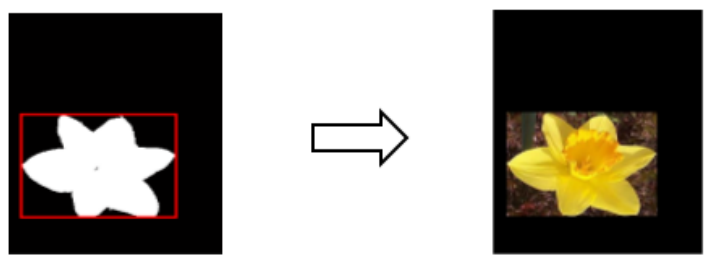

Figure 5 Segmented image

\subsection{Feature Extraction Techniques}

\subsubsection{GLCM}

Texture feature is extracted by using GLCM method. It is applied on grayscale image hence we have to convert the segmented color image into grayscale image. Texture feature calculations means to measure the variation in intensity at interested pixel in an image which can use the GLCM contents [1] [6]. Mainly two steps are used for the extraction of Co-occurrence texture features [1] [7]. First step is to create a GLCM by using spatial co-occurrences of pixels in pair, which is separated by a particular angle, and distance [1] [6] [13]. The GLCM is a square matrix of $\mathrm{M} \times \mathrm{M}$, where $\mathrm{M}$ is the different gray levels present in an image [1] [6]. In second step, computed GLCM is used to calculate the different property of GLCM like correlation, contrast, energy, local homogeneity, norm entropy, inverse difference moment, maximum probability, cluster prominence and cluster shade. Here we have used four features contrast, correlation energy and homogeneity [1] [6]. Formulas for feature extraction techniques are given below.

$$
\begin{aligned}
& \text { Contrast }=\sum_{i, j}|i-j|^{2} p(i, j) \\
& \text { Correlation }=\sum_{i, j} \frac{(i-\mu i)(j-\mu j) p(i, j)}{\sigma_{i} \sigma_{j}} \\
& \text { Energy }=\sum_{i, j} p(i, j)^{2} \\
& \text { Homogeneity }=\sum_{i, j} \frac{p(i, j)}{1+|i-j|}
\end{aligned}
$$

\begin{tabular}{|c|c|}
\hline Features & Values \\
\hline Contrast & {$\left[\begin{array}{lll}0.3858 & 0.3222\end{array}\right]$} \\
\hline Correlation & {$\left[\begin{array}{lll}0.9535 & 0.9612\end{array}\right]$} \\
\hline Energy & {$\left[\begin{array}{lll}0.4982 & 0.4993\end{array}\right]$} \\
\hline Homogeneity & {$\left[\begin{array}{lll}0.9317 & 0.9360\end{array}\right]$} \\
\hline
\end{tabular}

Table 1 Flower image representation in numeric value using GLCM method

\subsubsection{Color moment}

Color features can be extracted using Color Moment [14]. Central moments uniquely describe a probability distribution in the same way that color moments are measures that characterize color distribution in an image [14]. Color moments are mainly used for color indexing purposes. In various application like image retrieval, to compare the two images as how both are similar in terms of color then color feature is used [14]. Color moments are rotation and scaling invariant. Color moments give information about shape and color [14]. When lighting conditions are changing then color is a good feature to use, but they cannot handle the situation very successfully when part of an image is block by another [14]. It is computed for any color model like RGB, HSV, and CMYK. In color model, per 
channel, three color moments are computed. E.g. if color model is RGB then 9 moments and if color model is CMYK then 12 moments are computed [14].

a) Mean

The first color moment is mean. It can be interpreted as the average color in the image [14].

$$
E_{i}=\sum_{j=1}^{N} \frac{1}{N} p i j
$$

\section{b) Standard Deviation}

The second color moment is standard deviation, which can be calculated by taking the square root of the variance of the color distribution [14].

$$
\sigma_{i}=\sqrt{\left(\frac{1}{N} \sum_{j=1}^{N}(p i j-E i)^{2}\right)}
$$

Here in this system we used only two color moment for RGB color model i.e. mean and standard deviation. For R, G and B channels, values of mean and standard deviation are shown in table II. This value is only for one image.

Table 2 Flower image representation in numeric value using Color moment

\begin{tabular}{ccc}
\hline & Mean & Standard Deviation \\
\hline R & 49.3443 & 86.3025 \\
G & 40.3606 & 73.4733 \\
B & 11.8193 & 24.4136 \\
\hline
\end{tabular}

\subsection{Classification}

For classification, firstly the input feature vector is created which is extracted using GLCM [1] and color moment. The input feature vector consists of eight GLCM values and six Color moment values. This input vector is given to neural network for classification [1].

\section{Experimental Results}

The proposed system is tested for 5 flower class (Daffodil, Snowdrop, lily Valley, Crocus and Tiger lily) and 40 images from each class hence total 200 images is used for classification [13]. The number of neurons in hidden layer is 30 . The table III. Shows the accuracy of classification using GLCM method, color moment and combination of both. Accuracy of this system is $95.0 \%$ when both features are used.

Table 3 Accuracy of classification using different methods

\begin{tabular}{cc}
\hline Methods used & Accuracy \\
\hline GLCM (Texture feature) & $40.0 \%$ \\
Color moment (Color feature) & $65.0 \%$ \\
GLCM \& Color moment (Hybrid feature) & $95.0 \%$ \\
\hline
\end{tabular}

\section{Conclusion}

The flower classification system based on digital image processing takes the input image which is flower image taken from dataset. In this system of flower classification for speedy processing the input 
original flower image is resized. To acquire flower part in the image, which is foreground, threshold is used for segmentation. Texture feature and color feature are extracted by using GLCM and color moment respectively. For flower classification, neural network classifier is used. The accuracy of this flower classification system is $95.0 \%$. The accuracy of system can be improved by considering other features, such as edge and shape.

\section{References}

[1] Dr.S.M.Mukane and Ms.J.A.Kendule "Flower Classification Using Neural Network Based Image Processing," IOSR Journal of Electronics and Communication Engineering (IOSR-JECE), 2013, pp. $80-85$

[2] T. Tiay, P. Benyaphaichit and P. Riyamongkol, "Flower recognition system based on image processing," Student Project Conference (ICT-ISPC), 2014 Third ICT International, Nakhon Pathom, 2014, pp. 99-102.

[3] C. Y. Huang, Y. L. Lin and W. H. Hsu, "Augmenting flower recognition by automatically expanding training data from web," Multimedia Signal Processing (MMSP), 2015 IEEE 17th International Workshop on, Xiamen, 2015, pp. 1-6.

[4] S. W. Hong and L. Choi, "Automatic recognition of flowers through color and edge based contour detection," Image Processing Theory, Tools and Applications (IPTA), 2012 3rd International Conference on, Istanbul, 2012, pp. 141-146.

[5] M. E. Nilsback and A. Zisserman, "A Visual Vocabulary for Flower Classification," 2006 IEEE Computer Society Conference on Computer Vision and Pattern Recognition (CVPR'06), 2006, pp. 1447-1454.

[6] D S Guru, Y. H. Sharath and S. Manjunath, "Texture Features and KNN in Classification of Flower Images," Recent Trends in Image Processing and Pattern Recognition (RTIPPR), 2010

[7] Basavanna M and S. S. Gornale, "Identification and Classification of Flowers Images using Colour models," International Journal of Advanced Research in Computer Science and Software Engineering (IJARCSSE), 2015, pp. 1003-1007.

[8] Das, M., Manmatha, R., and Riseman, E. M. 1999. Indexing flower patent images using domain knowledge. IEEE Intelligent systems, Vol. 14, No. 5, pp. 24-33.

[9] A. Angelova, S. Zhu and Y. Lin, "Image segmentation for large-scale subcategory flower recognition," 2013 IEEE Workshop on Applications of Computer Vision (WACV), Tampa, FL, 2013, pp. 39-45.

[10] Y. Y. Boykov and M. P. Jolly. Interactive graph cuts for optimal boundary and region segmentation of objects in N-D images. In Proc. ICCV, volume 2, pages 105-112, 2001.

[11] Hui Yu, Mingjing Li, Hong-Jiang Zhang, Jufu Feng. "Color Texture Moments for ContentBased Image Retrieval". Proc. IEEE Intl Conf. on Image Processing, pp. 929-932, Sep. 2002

[12] F. Siraj, H. M. Ekhsan and A. N. Zulkifli, "Flower image classification modeling using neural network," 2014 International Conference on Computer, Control, Informatics and Its Applications (IC3INA), Bandung, 2014, pp. 81-86.

[13] http://www.robots.ox.ac.uk/ vgg/data/flowers/17/

[14] https://en.wikipedia.org/wiki/Color moments. 